\title{
Bronchoalveolar lavage fluid cytological findings in healthy Amiata donkeys
}

\author{
Valentina Vitale $^{1^{*}}$, Francesca Bonelli ${ }^{2,3}$, Angela Briganti ${ }^{2,3}$ and Micaela Sgorbini ${ }^{2,3}$ \\ ${ }^{1}$ Veterinary Teaching Hospital, Sydney School of Veterinary Science, The University of Sydney, Camden, Australia \\ ${ }^{2}$ Department of Veterinary Sciences, University of Pisa, Pisa, Italy \\ ${ }^{3}$ Veterinary Teaching Hospital "Mario Modenato", Department of Veterinary Sciences, University of Pisa, Pisa Italy
}

\begin{abstract}
Background: The approach to respiratory diseases in donkeys is similar to that for horses; nevertheless, Bronchoalveolar lavage fluid (BALF) and tracheal wash cytology in this species have been described only a few times in the literature. Aim: To describe BALF cytological findings in a cohort of 24 healthy Amiata donkeys.

Methods: Bronchoalveolar lavage (BAL) was carried out via standing sedation with a large animal where the BAL catheter passed blindly through the nasal passage into the trachea.

Results: The total nucleated cell count of the BALF was found similar to that already described in healthy horses and donkeys. No differences in the differential count were observed according to age and sex. A decreased macrophage percentage and an increased eosinophil percentage were observed in our donkey population when compared to the existing reference range for horses.

Conclusion: The reference intervals for BAL cytology in donkeys may be significantly different for those referred for horses.

Keywords: Donkey, BAL, Respiratory, Cytology.
\end{abstract}

\section{Introduction}

The domestic donkey (Equus asinus) is kept predominantly as a companion animal in the UK, European countries, and North America, but is frequently used as a working animal in developing countries worldwide (Burden et al., 2016). When dealing with a donkey's respiratory disease, it has been stated that clinicians should approach the case in a similar manner as for horses (Thiemann, 2012). Nevertheless, donkeys present some anatomical and physiological differences from horses and may be more or less severely affected by the same pathogens. Owing to their stoic nature and relatively insensitive cough reflex (Thiemann, 2012; Rickards and Thiemann, 2019), subtle changes in the early stages of disease progression may go unnoticed. If we consider also their non-athletic lifestyle, some diseases may appear relatively late and at a more severe stage when compared to horses, carrying a poorer prognosis (Rickards and Thiemann, 2019).

Tracheal wash (TW) or bronchoalveolar lavage (BAL) cytology results are considered useful for the investigation of lower airway respiratory disease (Barrandeguy and Carrossino, 2018). The choice of the sampling method is related to the suspected disease process (Rossi et al., 2018). TW is an aseptic percutaneous technique that is preferred when an infectious disease is suspected because secretions from the affected lung areas will be collected in the trachea (Couetil and Hawkins, 2013). Bronchoalveolar lavage fluid (BALF) cytology is recommended in suspected non-bacterial lung disease, and it is considered to be a more sensitive technique for detecting lower airway inflammation (Tilley et al., 2013; Couetil et al., 2016; Barrandeguy and Carrossino, 2018). BAL and TW procedures in donkeys have been described only a few times in the literature (Delvaux et al., 2001; Campi et al., 2008; Shawaf, 2019).

This study aimed to describe BAL cytological findings in a cohort of healthy Amiata donkeys.

\section{Material and Methods}

\section{Animals}

A cohort of 24 healthy Amiata donkeys, 12 jennies and 12 jacks, aged between 2 and 17 years and owned by the Regional Stud Farm (Tuscany, Italy) were enrolled in this study. The jennies' herd was housed in a large sand paddock with two shelters, while jacks were housed in single boxes $4 \times 4$ m opened to a small single paddock. They were fed a diet consisting of ad libitum grass hay and were vaccinated against tetanus, influenza, and herpesvirus- 1 and -4 , once per year. All the animals were submitted to weekly physical examinations, and to be included in the study, where they should have maintained a normal physical exam and thoracic and tracheal auscultation for at least 3 months prior to the experimental procedure and also in the following 3 months. All donkeys were routinely dewormed with Ivermectin (Equalan, Boehringer Ingelheim Animal Health Italia S.p.a.) with a dose of $0.2 \mathrm{mg} / \mathrm{kg}$ orally, at 6-month intervals. The night before the experimental 
Table 1. Median, minimum, and maximum values of BALF cytological characteristics in 24 healthy Amiata donkeys with reference ranges of horses.

\begin{tabular}{ccccccc}
\hline & TNCC cells/ml & $\begin{array}{c}\text { Macrophages } \\
(\mathbf{\%})\end{array}$ & $\begin{array}{c}\text { Lymphocytes } \\
(\mathbf{\%})\end{array}$ & $\begin{array}{c}\text { Neutrophils } \\
(\mathbf{\%})\end{array}$ & $\begin{array}{c}\text { Eosinophils } \\
(\mathbf{\%})\end{array}$ & $\begin{array}{c}\text { Mast cells } \\
(\mathbf{\%})\end{array}$ \\
\hline Donkeys & $200(100-700)$ & $38.5(16-66)$ & $45(5-70)$ & $3(0-22)$ & $2(0-62)$ & $0(0-3)$ \\
$\begin{array}{c}\text { Reference } \\
\text { ranges (horses) }\end{array}$ & $<300$ & $55-65$ & $28-38$ & $<10$ & $<1$ & $<2$ \\
\hline
\end{tabular}

procedures, the donkeys were housed in pairs within $4 \times 4 \mathrm{~m}$ boxes with a cement floor and no bedding. The housing in groups of two was chosen to avoid unnecessary stress for the animals. Food, but not water, was withheld for 8-12 hours.

\section{Experimental procedure}

The study took place between January and March 2014. The donkeys received an intramuscular injection with detomidine (Detogesic, Zoetis Italia s.r.l., Roma, Italy; $60 \mu \mathrm{g} / \mathrm{kg}$ ) and butorphanol (Nargesic, ACME s.r.l., Cavriago, Italy; $50 \mu \mathrm{g} / \mathrm{kg}$ ) while being housed in their boxes and immediately transferred to an examination room later on. Each subject was entered in a stock and the BAL was carried out with a large animal BAL catheter (Bivona Inc., Gary, IN) of $3 \mathrm{~m}$ length, $10 \mathrm{~mm}$ of inner diameter, with an inflatable cuff. The tube was passed blindly through the nasal passage and then moved caudally into the trachea. $20 \mathrm{ml}$ syringes were prefilled with a solution of lidocaine (Lidocaina 2\%, Zoetis Italia s.r.l., Roma, Italy) diluted at $0.66 \%$ with a $0.9 \%$ saline solution that was used to desensitize the tracheal lumen while advancing the tube for antitussive purposes. When the BAL tube reached the third- to fourth-generation bronchi, the cuff was inflated with air and $300 \mathrm{ml}$ of warm, sterile, isotonic, and crystalloid solution was infused to sample a large area of the lung and avoid disparities in the evaluation of cells differential count (Hoffman, 2008). Samples were collected with gentle, but constant manual aspiration using $60 \mathrm{ml}$ syringes. When at least $40 \%$ of the infused volume was recovered (Ainsworth, 2010), the cuff was deflated and the tube was removed.

BALF was collected in ethylenediaminetetraacetic acid tubes, to reduce cell clumping and processed within 1 hour from the collection. No fixatives (e.g., formalin or alcohol) were used to avoid altering cell morphology (Hoffman, 2008).

\section{Cytological analysis}

The total nucleated cell count (TNCC) was carried out on BALF samples with an automated counter(Hecovet, SEAC-RADIM Co, Florence, Italy). Then, the samples were spun down for 10 minutes, and the supernatant was poured off. The remaining sample was mixed until it looked homogeneous by gently flicking the bottom of the tubes and used for slide preparation by cytocentrifugation (Cytofuge 2, Statspin, Westwood, MA; $400 \mathrm{ml}$ per slide and $300 \mathrm{~g}$ rpm for 10 minutes). The slides were air-dried using a fan and stained with the May-Grunwald Giemsa stain using an automatic slide stainer (Aerospray Hematology Slide Stainer mod. 7150, Delcon, Italy). The staining time was 12 minutes. No media was used to mount the slides and no toluidine blue stain was used for the identification of metachromatic cells. A single 10-year experienced blinded veterinarian carried out the differential cell counts. Initially, the stained smears were scanned at low magnification $(\times 200)$, then at higher magnification $(\times 400)$ to reach an optimal monolayer area. Then, the cells were recognized and counted with oil immersion $(\times 1,000)$. The technique of scanning the stained smear started from the upper edges and then continued to the center until arriving at the lower edges to avoid scanning the already seen areas. Four hundred cells were counted per each differential count, using a bright-field light microscopy.

\section{Statistical analysis}

Data were analyzed for distribution using the Shapiro-Wilk test. Since data showed a non-Gaussian distribution, the results were expressed as median, minimum, and maximum values.

The Mann-Whitney test to compare ranks was applied to verify differences in the TNNC and differential cell count between female vs male donkeys and between young ( $<$ or equal to 7 years) and mature ( $>$ to 7 years) donkeys (Ainsworth, 2010).

Statistical significance was set at $p<0.05$. Statistical analysis was carried out using a commercial software (Graph Pad Prism, San Diego, CA).

\section{Ethical approval}

Ethical approval ( ${ }^{\circ}$ 9069/2014) by the Ethics Committee on Animal Experimentation of the University of Pisa and the owner's written consent were obtained.

\section{Results}

Sixteen of the 24 donkeys were simultaneously enrolled in another previously conducted study (Evangelista et al., 2018).

The results on the TNCC values and percentage of different inflammatory cells of the BAL are presented in Table 1 as median, minimum, and maximum values together with the reference intervals described for horses (McKenzie, 2008). Statistically significant differences related to sex were observed for TNNC ( $p=0.0334)$, but not for the differential cell count $(p$ $=0.8761,0.6594,0.1148,0.8073$, and $>0.9999$ for macrophages, lymphocytes, neutrophils, eosinophils, and mast-cells, respectively). In particular, male 
donkeys showed higher values of TNNC ( $200 \mathrm{cell} / \mathrm{ul})$ than the female ones $(100 \mathrm{cell} / \mathrm{ul})$.

No statistically significant differences were observed related to age (18 young vs 6 old donkeys) for any of the BALF parameters analyzed $(p=0.0815,0.4240$, $0.9332,0.9590,0.6590$, and 0.0543 for TNNC, macrophages, lymphocytes, neutrophils, eosinophils, and mast-cells, respectively).

\section{Discussion}

This study describes the cytological findings of the BALF in 24 healthy Amiata donkeys. With such a globally ubiquitous species, the relevance of published reference intervals to local populations is important, thus these intervals may not be relevant to other donkey populations, such as working donkeys in tropical regions. The Amiata donkey originated from Tuscany, in central Italy, and takes its name from the Monte Amiata in the province of Grosseto. To date, it is especially diffused throughout Tuscany and Liguria and has been particularly used for tourism and pet therapy.

BAL procedure is frequently carried out during the investigation of lower airway diseases; nevertheless, results are often interpreted by comparing them with reference values for horses (Mendoza et al., 2018). Two previous studies described BALF cytological findings in healthy donkeys (Campi et al., 2008; Shawaf, 2019); nevertheless, the number included in each study was limited and the populations involved came from completely different countries, so important differences in environment can exist between the two papers. While Campi et al. (2008) described a slight increase in macrophages' percentage when compared with horses, Shawaf (2019) reported a decreased proportion of macrophages and a higher percentage of eosinophils when comparing the results with the ranges for horses. In our population, the TNNC of the BALF was similar to what has already been described in healthy horses (Pacheco et al., 2014; Mendoza et al., 2018) and donkeys (Campi et al., 2008; Shawaf, 2019). In agreement with what has already been described in horses, no differences in the differential count were observed according to the age and sex of the subjects (Porto Sad et al., 2013; Pacheco et al., 2014). The reason for the difference in TNNC between males and females is unclear, and it is possibly a type II error caused due to the small sample size.

In agreement with both previous studies (Campi et al., 2008; Shawaf, 2019), we found a slightly decreased macrophages' percentage and an increase in eosinophils' percentage when comparing these values with those of the horses (McKenzie, 2008; Pacheco et al., 2014; Couetil et al., 2016). A mean of $2 \%$ eosinophils was observed in our population but high variability existed between single subjects with a range between $0 \%$ and $62 \%$ (Standard deviation: 20.33). Several hypotheses may explain this eosinophilia in the BALF of the donkeys in this report. First of all, donkeys can host a high numbers of adult lungworms (Dictyocaulus arnfieldi), showing minimal to no clinical signs (Thiemann, 2012). Although the population included in this study was subjected to periodical deworming treatments with Ivermectin and no larvae were observed at the BALF cytology, it is not possible to completely rule out lung parasitosis. The treatment of choice for lungworms is macrocyclic lactones (Thiemann and Bell, 2001) but resistance to this anthelmintic class may exist, thus the treatment in the present herd would not have been effective. Another possibility is that, at the time of the experiment, donkeys got a new infestation, although, due to the lack of introduction of new subjects in the herd, this is less likely.

An increase in eosinophils' percentage in the BALF cytology is also described in mild equine asthma. Horses with this condition usually show few clinical signs that include poor performance and chronic occasional coughing (Couetil et al., 2016). Nevertheless, the animals included in this study did not perform any athletic activity and donkeys, in general, have poor cough reflex (Thiemann and Bell, 2001); thus, these two factors together could have contributed to the normal physical examinations recorded prior and after the study. Less likely explanations of the eosinophilia are neoplastic or immune-mediated processes (Morrow et al., 2010); nevertheless, these rarely affect more than a few subjects in a herd.

Idiopathic pulmonary fibrosis is a common condition in donkeys and is often reported as an incidental finding at the post-mortem examination, despite the severe macroscopic alterations (Miele et al., 2014). Although its etiology has not been exactly defined, the chronic nature of the pathology would suggest a progressive and ongoing irritation of the lungs. This may be consistent with an allergen-induced hypersensitivity or repeated toxin-induced damage (Morrow et al., 2010). As donkeys can cope well with reduced lung function (Thiemann, 2012), the respiratory disease may be difficult to identify due to their stoicism, relatively insensitive cough reflex and the lack of athletic activity (Thiemann and Bell, 2011; Thiemann, 2012). Therefore, undetected pulmonary diseases may progress silently over time. Thus, screening of healthy subjects with a more invasive procedure, such as a BAL, may be advisable to avoid the development of pulmonary fibrosis.

Based on the findings of this study, it is unclear if a higher percentage of eosinophils in the BALF of donkeys should be interpreted as normal or if, due to the stoic nature of this animal, it may be the only alteration present in course of a mild lower respiratory airway disease.

A limitation of this study is that, with the stain used, mast cells could have been underestimated as toluidine 
blue is required as an additional stain for better sensitivity in the detection of mast cells (Ainsworth, 2010; Cian et al., 2015).

Further studies with an increased number of subjects and from different environments should be carried out to establish accurate reference ranges for BALF cytology in this species. To correctly identify healthy subjects, pulmonary function tests, thoracic ultrasonography, and radiography may be associated with the physical examination. The Horse Owner Assessed Respiratory Signs Index (HOARSI) is a scoring system introduced to grade asthma severity in horses (Ramseyer et al., 2007). More completed scoring systems that take into account also endoscopic examination, BALF cytology, thoracic X-rays, arterial blood gas analysis, and lung function tests have been described in horses (Tilley et al., 2012; Simoes et al., 2019). All these staging systems may be applied to donkeys to identify mild or latent forms of respiratory diseases in this species.

\section{Conclusion}

As already found for blood parameters, the reference intervals for respiratory fluid cytology in donkeys may be significantly different for those generated for horses. The differences in the BAL fluid cytology noted in this study highlight the importance of using species-appropriate reference intervals for clinical assessment.

\section{Acknowledgements}

The authors wish to thank the Regional Stud Centre of Tuscany, Italy. This work was supported by funds of the University of Pisa.

\section{References}

Ainsworth, D.M. 2010. Review of recurrent airway obstruction (RAO, Heaves): diagnosis and treatment options. In of the 12th International congress of the World Equine Veterinary Association, pp 100-106.

Barrandeguy, M.E. and Carossino, M. 2018. Infectious diseases in donkeys and mules: an overview and update. J. Equine Vet. Sci. 65, 98-105.

Burden, F.A., Hazell-Smith, E., Mulugeta, G., Patrick, V., Trawford, R. and Brooks Brownlie, H.W. 2016. Reference intervals for biochemical and haematological parameters in mature domestic donkeys (Equus asinus) in the UK. Equine Vet. Educ. 28(3), 134-139.

Campi, C., El Mohaine, B., Lamrini, H. and Crane, M. Working mules and donkeys: preliminary observations on videoendoscopic anatomy of the upper airway tract and BAL cytology. In Proceedings of the 10th International congress of the World Equine Veterinary Association, 2008, pp 548-549.

Cian, F., Monti, P. and Durham, A. 2015. Cytology of the lower respiratory tract in horses: an updated review. Equine Vet. Educ. 27(10), 544-553.
Couetil, L.L. and Hawkins, J. 2013. Diagnostic tests and therapeutic procedures. In Respiratory diseases of the horse. Eds., Couetil, L.L. and Hawkins, J. London, UK: Manson Publishing Ltd, pp: 55-58.

Couetil, L.L., Cardwell, J.M., Gerber, V., Lavoie, J.-P., Léguillette, R. and Richard, E.A. 2016. Inflammatory airways disease of horses - revised consensus statement. J. Vet. Intern. Med. 30, 503-515.

Delvaux, V., Kirschvink, N., Amory, H., Busoni, V., Art, T. and Lekeux, P. 2001. Spécificité de la function cardiorespiratoire de l'ane (Equus asinus). Prat. Vét. Equine 33, 21-28.

Evangelista, F., Tayari, H., Degani, M., Nocera, I. and Briganti, A. 2018. Sedative and respiratory effects of intramuscular detomidine and butorphanol in donkeys sedated for bronchoalveolar lavage. J. Equine Vet. Sci. 69, 96-101.

Hoffman, A.M. 2008. Bronchoalveolar lavage: sampling technique and guidelines for cytologic preparation and interpretation. Vet. Clin. Equine 24, 423-435.

McKenzie, H. 2008. Transtracheal and bronchoalveolar fluid analysis. In The equine hospital manual. Eds. Corley, K. and Stephen, J.E. Oxford, UK: Blackwell Publishing Ltd., appendix 681.

Mendoza, F.J., Toribio, R.E. and Perez-Ecija, A. 2018. Donkey Internal Medicine - part II: cardiovascular, respiratory, neurologic, urinary, ophthalmic, dermatology, and musculoskeletal disorders. J. Equine Vet. Sci. 65, 86-97.

Miele, A., Dhaliwal, K., Du Toit, N., Murchison, J.T., Dhaliwal, C., Brooks, H., Smith, S.H., Hirani, N., Schwarz, T., Haslett, C., Wallace, W.A. and McGorum, B.C. 2014. Chronic pleuropulmonary fibrosis and elastosis of aged donkeys. Chest 145(6), 1325-1332.

Morrow, L.D., Smith, K.C., Piercy, R.J., du Toit, N., Burden, F.A., Olmos, G., Gregory, N.G. and Verheyen, K.L.P. 2010. Retrospective analysis of post-mortem findings in 1444 aged donkeys. J. Comp. Pathol. 144, 145-156.

Pacheco, A.P., Paradis, M.R., Hoffman, A.M., Hermida, P., Sanchez, A., Nadeau, J.A., Tufts, M. and Mazan, M.R. 2014. Age effects on blood gas, spirometry, airway reactivity, and bronchoalveolar lavage fluid cytology in clinically healthy horses. J. Vet. Intern. Med. 28, 603-608.

Porto Sad, E., de Alencar, N.A., Viscardi, V., de Mello Costa, M.F., Hess, T.M. and Barroso Lessa, D.A. 2013. Cytology profile and age influence in the equine bronchoalveolar lavage in healthy and asymptomatic inflammatory airway disease. Cienc. Rural 43(3), 452-455.

Ramseyer, A., Gaillard, C., Burger, D., Straub, R., Jost, U., Boog, C., Marti, E. and Gerber, V. 2007. Effects of genetic and environmental factors on chronic lower airway disease in horses. J. Vet. Intern. Med. $21,149-156$. 
Rickards, K.J. and Thiemann, A.K. 2019. Respiratory disorders of the donkey. Vet. Clin. Equine 35, 561-571.

Rossi, H., Virtala, A.M., Raekallio, M., Rahkonen, E., Rajamaki, M.M. and Mykkanen, A. 2018. Comparison of tracheal wash and bronchoalveolar lavage cytology in 154 horses with and without respiratory signs in a referral hospital over 2009-2015. Front. Vet. Sci. 5, 61; doi:10.3389/fvets.2018.00061

Shawaf, T. 2019. Cytological analysis of tracheal washing and bronchoalveolar lavage fluid obtained from donkeys with chronic lung disease. Alexandria J. Vet. Sci. 60(1), 15-21.

Simoes, J., Sales Luis, J.P. and Tilley, P. 2019. Contribution of lung function tests to the staging of severe equine asthma syndrome in the field. Res. Vet. Sci. 123, 112-117.

Thiemann, A.K. 2012. Respiratory disease in the donkey. Equine Vet. Educ. 24(9), 469-478.
Thiemann, A.K. and Bell, N.J. 2001. The peculiarities of donkey respiratory disease. In Equine respiratory diseases. (Lekeux P., ed.). Ithaca, NY, International Veterinary Information service (www.ivis.org), pp: 1101-1118.

Tilley, P., Sales Luis, J.P. and Branco Ferreira, M. 2012. Correlation and discriminant analysis between clinical, endoscopic, thoracic x-ray and bronchoalveolar lavage fluid cytology scores, for staging horses with recurrent airway disease (RAO). Res. Vet. Sci. 93, 1006-1014.

Tilley, P., Simoes, J. and Sales Luis, J.P. 2013. Preliminary results of the use of bronchoalveolar lavage (BAL) for the diagnosis of lower airways disorders in donkeys. 5th World Equine Airway Symposium abstract book, 15-17th July, 2013, Calgary, Canada. 